\title{
Gate Semi-Around Si Nanowire FET Fabricated by Conventional CMOS Process with Very High Drivability
}

\author{
Soshi Sato ${ }^{1}$, Yeonghun Lee ${ }^{1}$, Kuniyuki Kakushima ${ }^{2}$, Parhat Ahmet ${ }^{1}$, \\ Kenji Ohmori ${ }^{3}$, Kenji Natori ${ }^{1}$, Keisaku Yamada ${ }^{3}$ and Hiroshi Iwai ${ }^{1}$ \\ ${ }^{1}$ Frontier Research Center, ${ }^{2}$ Interdisciplinary Graduate School of Science and Engineering, \\ Tokyo Institute of Technology, 4259, Nagatsuta, Midori-ku, Yokohama 226-8502, Japan \\ ${ }^{3}$ Nanotechnology Laboratory, Waseda University, 513, Waseda-Tsurumaki, Shinjuku-ku, Tokyo 162-0041, Japan
}

\begin{abstract}
Gate semi-around silicon nanowire (SiNW) FETs have been fabricated and their electrical characteristics, especially on the drivability, have been assessed for future high performance devices. Among different wire size, a SiNW FET with a cross-section of $12 \times 19 \mathrm{~nm}^{2}$ has shown an improvement in the on-current $\left(I_{\mathrm{ON}}\right)$ when normalized by the channel peripheral length. A high $I_{\mathrm{ON}}$ over $1600 \mu \mathrm{A} / \mu \mathrm{m}$ at an overdrive voltage of 1 $V$ has been achieved with a gate length and an oxide thickness of 65 and $3 \mathrm{~nm}$, respectively. The origin of the high drivability has been speculated by higher carrier density, improved carrier mobility and the reduction in the series resistance.
\end{abstract}

\section{INTRODUCTION}

Performance improvements by downsizing the CMOS technology have required new structures, including ultra-thin SOI and double-gate devices, to overcome the severe short channel effects (SCE), which induces an increase in off-state leakage current $\left(I_{\mathrm{OFF}}\right)$ to degrade the on-off ratio. Recently, silicon nanowire (SiNW) FETs have attracted much attention as they enable strong channel potential controllability by the gate electrode [1]. Beside, improvements in on-current $\left(I_{\mathrm{ON}}\right)$ with SiNW FETs have been reported, which are advantageous for high-speed with low power consumption application [2]. In this paper, gate semi-around SiNW FETs have been fabricated using conventional CMOS processes and their electrical characteristics, especially on the drivability, have been assessed for future high performance devices.

\section{DEVICE FABRICATION PROCESS}

A (100)-oriented SOI wafer was used as a starting material with an SOI layer and a buried oxide thickness of 75 and $50 \mathrm{~nm}$, respectively. SiNWs were formed by oxidation of fin patterns with a Si-nitride mask atop. Rectangular-like shape SiNWs with a wire height $\left(h_{\mathrm{NW}}\right)$ of $12 \mathrm{~nm}$ and wire widths $\left(w_{\mathrm{NW}}\right)$ of 19,28 and $39 \mathrm{~nm}$ were fabricated. The Si-nitride mask suppresses the excess thinning of the embedded source and drain (S/D) pad regions to avoid any unexpected increase in the series resistance $\left(\mathrm{R}_{\mathrm{SD}}\right)$. After striping the nitride and the formed oxides, a conventional self-aligned gate stack formation including gate oxidation and poly-Si deposition was conducted. The gate oxide thickness was set to $3 \mathrm{~nm}$.
Phosphorus ion implantations (boron for $\mathrm{pFET}$ ) were chosen for extension formation with doses of $1 \times 15 \mathrm{~cm}^{-2}$ at $15 \mathrm{keV}$ and $5 \times 15 \mathrm{~cm}^{-2}$ at $5 \mathrm{keV}$ for S/D region. After S/D activation annealing, a nickel self-aligned silicidation process was applied to reduce the $\mathrm{R}_{\mathrm{SD}}$. The key process is summarized in figure 1 and the detailed processes to fabricate semi gatearound SiNW FETs are shown in ref [3]. The SEM and TEM images of the fabricated SiNW FET are shown in figure 2.

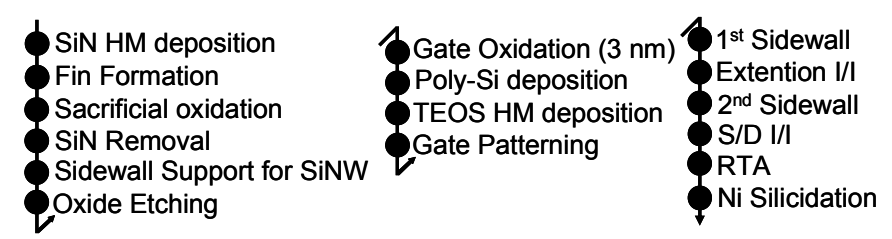

Figure 1. The key process flow for SiNW FETs using CMOS compatible process.

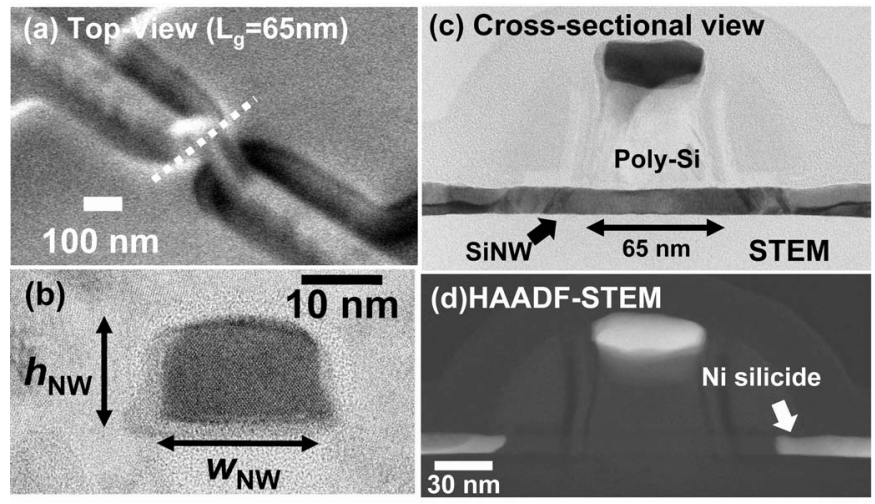

Figure 2. Typical SEM and TEM images of the fabricated SiNW FET.

\section{ELECTRICAL CHARACTERISTICS}

The dc-characteristics $\left(I_{\mathrm{d}}-V_{\mathrm{d}}\right.$ and $I_{\mathrm{d}}-V_{\mathrm{g}}$ curves $)$ of the smallest ( $h_{\mathrm{NW}}$ of $12 \mathrm{~nm}$ and $w_{\mathrm{NW}}$ of $19 \mathrm{~nm}$ ) SiNW FETs with a gate length and gate oxide of 65 and $3 \mathrm{~nm}$, respectively, are shown in figure 3. A well behaved transistor operation was confirmed for both $n$ - and $p$-FET. The on-current of $n$-FET under an overdrive and a drain voltage of $1 \mathrm{~V}$ showed a large 
value of $60 \mu \mathrm{A}$, whereas that of p-FET was as low as $22 \mu \mathrm{A}$. The low on-current of pFET is mainly due to large $\mathrm{R}_{\mathrm{SD}}$ and still needs process optimization. A fairly nice on-off current ratio of $>10^{6}$ with a DIBL and a favorable SS of $62 \mathrm{mV} / \mathrm{V}$ and $70 \mathrm{mV} / \mathrm{dec}$., respectively have been obtained, owing to the large channel potential control of the gate electrode. The smaller $w_{\mathrm{NW}}$ was, the better SCE immunity represented by $\mathrm{V}_{\text {th }}$ roll-off and SS was obtained, which indicate the advantages of small cross section against the SCE, which is in good agreement with previous reports [4]. Moreover, SS of 62-63 $\mathrm{mV} / \mathrm{dec}$. with $L_{\mathrm{g}}$ over $150 \mathrm{~nm}$ was obtained which strongly suggests that the density of surface states $\left(D_{i t}\right)$ is negligible with a rectangular-like shape $\mathrm{SiNW}$.
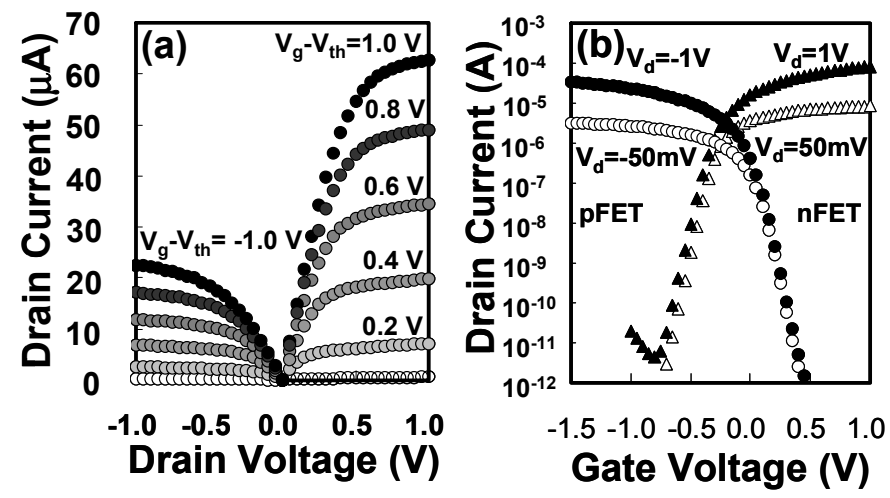

Figure 3. Dc characteristics of the fabricated gate semi-around SiNW FET $\left(\mathrm{h}_{\mathrm{NW}}=12 \mathrm{~nm}, \mathrm{w}_{\mathrm{NW}}=19 \mathrm{~nm}\right)$ with $\mathrm{L}_{\mathrm{g}}=65 \mathrm{~nm}$ and $\mathrm{T}_{\mathrm{ox}}=3 \mathrm{~nm}$.

The on-current per wire and on-current normalized by the channel peripheral width $\left(I_{\mathrm{ON}}\right)$ of the SiNW FETs with three different $w_{\mathrm{NW}}$ were plotted against the effective gate length $\left(L_{\text {eff }}\right)$ in figure 4. A large on-current per wire can be obtained by wider SiNW mostly owing to the larger channel peripheral width. However, when normalized with channel peripheral length, the $I_{\mathrm{ON}}$ showed large improvements with smaller size. As the only difference among the three SiNW is the $w_{\mathrm{NW}}$, one can speculate the effect of the rounded corner of the channel for $I_{\mathrm{ON}}$ improvement. Moreover, the $I_{\mathrm{ON}}$ showed an increasing trend with $L_{\text {eff }}$ scaling, indicating further improvement in the drivability by $L_{\mathrm{g}}$ scaling.
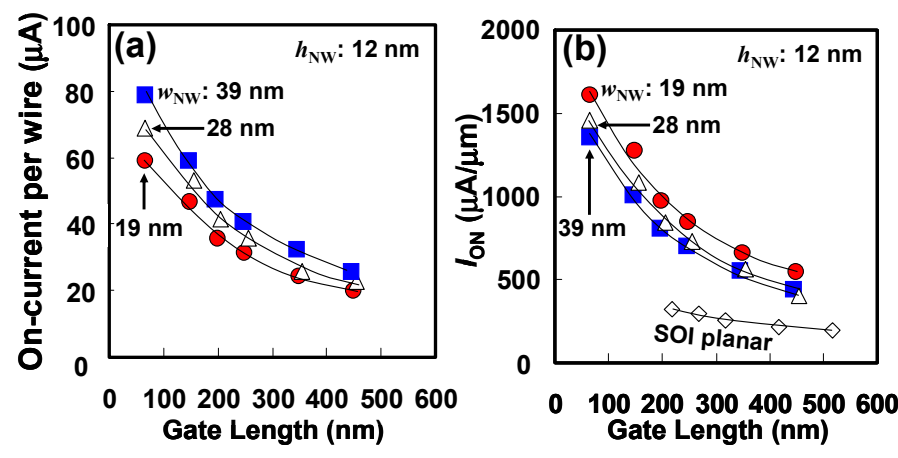

Figure 4. (a) On-current per wire and (b) $I_{\mathrm{ON}}$ normalized by peripheral channel width of three SiNW FET. The $I_{\mathrm{ON}}$ of planer SOI FETs fabricated on the same wafer are plotted as references.
To examine the drivability of SiNW FETs, planer SOI FETs with $\mathrm{T}_{\mathrm{Si}}$ of $28 \mathrm{~nm}$ were fabricated simultaneously on the same wafer and are shown in the figure as references. One can observe improved $I_{\mathrm{ON}}$ by more than 2 times with SiNW FET, especially with the smallest cross-section.

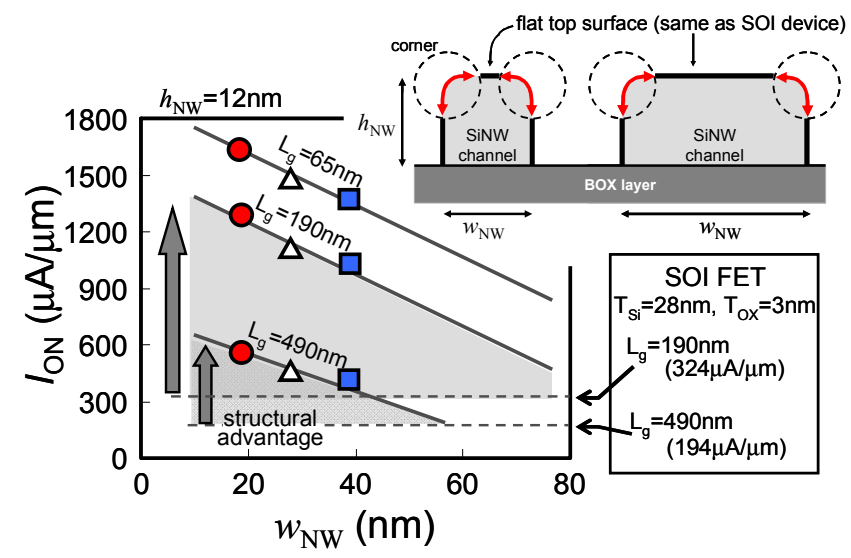

Figure 5. Structural advantage of SiNW FETs over SOI FETs with smaller $w_{\mathrm{NW}}$ for high drivability.

The effect of $w_{\mathrm{NW}}$ for SiNW FETs on the $I_{\mathrm{ON}}$ drivability is shown in figure 5. Compared to the planar SOI FETs with the

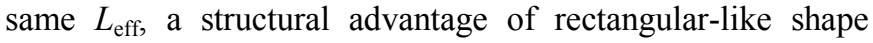
SiNW is more pronounced at smaller $w_{\mathrm{NW}}$. Note that the existence of multiple $V_{\text {th }}$ may be the concern with the rounded corners, however, no kink effect in the subthreshold characteristics was observed with our devices due to lowdoped channel [5].

The $I_{\mathrm{ON}}-I_{\mathrm{OFF}}$ characteristics measured from $L_{\text {eff }}$ of 500 to $65 \mathrm{~nm}$ are shown in figure 6 . Here the $I_{\mathrm{OFF}}$ is defined as the drain current normalized by the peripheral channel width at an overdrive voltage of $-0.3 \mathrm{~V}$ and a drain voltage of $1 \mathrm{~V}$. Although little difference is observed at large $L_{\text {eff }}$ region, a distinct improvement on the on-off ratio is obtained with smaller cross-section when $L_{\text {eff }}$ scaling is conducted. A large $I_{\mathrm{ON}}$ over $1600 \mu \mathrm{A} / \mu \mathrm{m}$ at an $I_{\mathrm{OFF}}$ of $1 \mathrm{nA} / \mu \mathrm{m}$ was achieved with a SiNW FET of $12 \times 19 \mathrm{~nm}^{2}, L_{\text {eff }}=65 \mathrm{~nm}$, and $T_{\mathrm{ox}}=3 \mathrm{~nm}$.

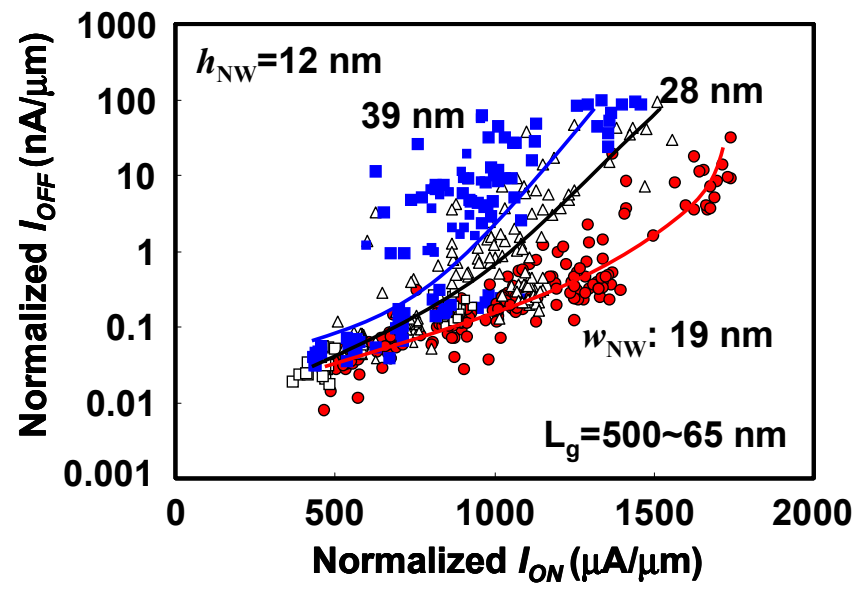

Figure 6. $I_{\mathrm{ON}}-I_{\mathrm{OFF}}$ characteristics of SiNW with different $w_{\mathrm{NW}}$. 
To analyze the origin of the large drivability of $I_{\mathrm{ON}}$ with SiNW FETs, the effective electron mobility $\left(\mu_{\text {eff }}\right)$ were extracted by SiNW FETs with multi-wire channel $(\mathrm{N}=64)$ using advanced split-CV method, which are shown in figure 7. To avoid any unexpected parasitic effects for gate-to-channel capacitance measurements, an advanced split-CV method was adopted using two different FETs; gate mask length of 550 and $250 \mathrm{~nm}$ [6]. The inversion carrier density $\left(\mathrm{N}_{\mathrm{s}}\right)$ was calculated using the sum of peripheral channel width of the SiNW obtained from TEM images. A large $\mu_{\text {eff }}$ peaking at $452 \mathrm{~cm}^{2} / \mathrm{Vs}$ and $405 \mathrm{~cm}^{2} / \mathrm{Vs}$ at $\mathrm{N}_{\mathrm{s}}$ of $10^{13} \mathrm{~cm}^{-2}$ was extracted with a SiNW of $12 \times 19 \mathrm{~nm}^{2}$. Compared with the $\mu_{\text {eff }}$ of the planer SOI FET, a large improvement especially at high $\mathrm{N}_{\mathrm{s}}$ can be obtained, presumably due to lower vertical electric field to the nanowire channel. The $\mu_{\text {eff }}$ showed little dependency on the $w_{\mathrm{NW}}$, indicating small surface roughness at the top of SiNW. However, a large degradation in $\mu_{\text {eff }}$ was observed when a large $h_{\mathrm{NW}}$ was designed, especially at high $\mathrm{N}_{\mathrm{s}}$ region where roughness scattering dominates. The reason might be the increase in the area of the etched side-surface to degrade the surface morphology so that process optimization including damage free plasma may improve the performance [7].

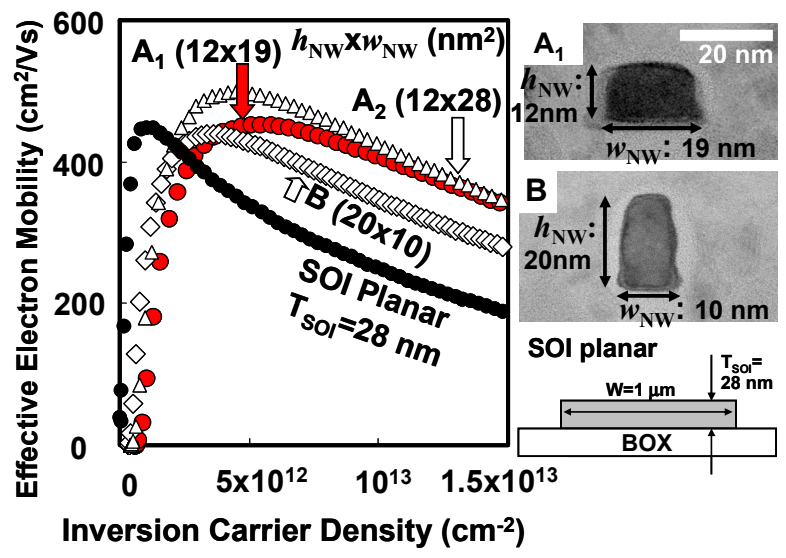

Figure 7. $\mu_{\text {eff }}$ of SiNW FETs obtained from advanced split-CV method.

The $\mathrm{R}_{\mathrm{SD}}$ was extracted using the above devices based on Chern's method. A small $R_{\mathrm{SD}}$ of $1.5 \mathrm{k} \Omega$ was obtained irrespective of the $w_{\mathrm{NW}}$. (fig. 8) This $\mathrm{R}_{\mathrm{SD}}$ value corresponds to only $10 \%$ of the total channel resistance $\left(\mathrm{R}_{\text {tot }}\right)$ for $L_{\text {eff }}$ of $65 \mathrm{~nm}$, owing to the process optimization for S/D formation. Note that As implantation instead of $\mathrm{P}$ resulted in 10 times higher $\mathrm{R}_{\mathrm{SD}}$, presumable due to the damages in the S/D regions as well as the difference in the Ni silicide formation [8].

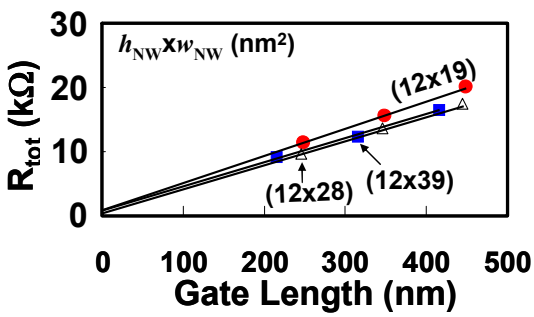

Figure 8. Extracted $\mathrm{R}_{\mathrm{SD}}$ of the SiNW nFETs usning Chern's method.
Another reason to enhance the drivability of the SiNW FETs might be the distribution in the carrier concentration. Here, a two-dimensional simulation to extract the inversion carrier concentration distribution profile in the SiNW channel were carried out using a Taurus device simulator under modified local density approximation (MLDA) method [9]. The simulated device was designed to have the same cross sectional shape as was obtained experimentally. The work function of the metal electrode was set to $4.1 \mathrm{eV}$ and a nondoped channel was assumed. Figure 9 shows the carrier concentration profile under a gate overdrive voltage of $1 \mathrm{~V}$. High density regions can be observed near the corners of the channels, owing to the electrostatics at the rounded surface and one can see that large amount of carriers located within the SiNW away from the surface. As the $w_{\mathrm{NW}}$ decreases, the high-density regions at the corners approach, so that the portion of the high-density region within the cross section increases. Line profiles at the corner and at the top planer regions showed an increase in the carrier concentration by 2.5 times at the corner for both structures, indicating advantage for higher drivability at corners.

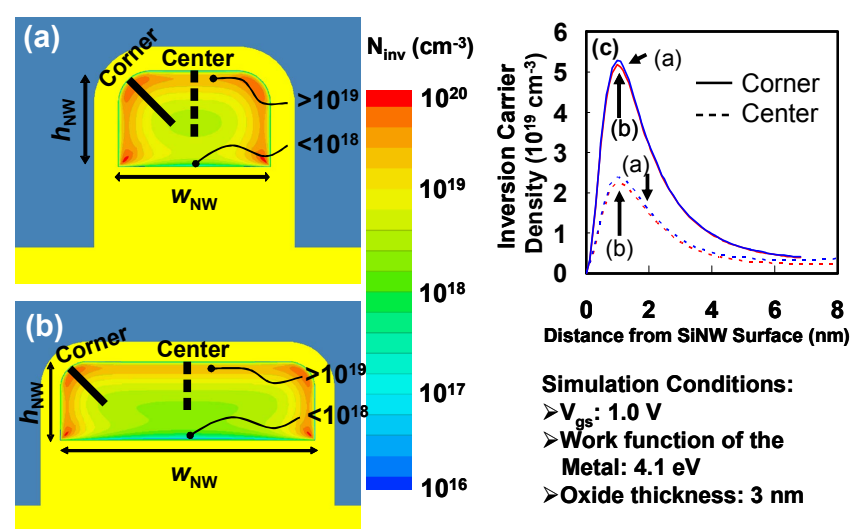

Figure 9. Two-dimensional simulation of the inversion carrier concentration within the SiNW chanels of (a) $12 \times 19 \mathrm{~nm}^{2}$ and (b) $12 \times 38 \mathrm{~nm}^{2}$. (c) Line profile of the carrier concentration at the top planar and at the coner.

Based on the above characterizations, the improvement in the drivability from SOI to SiNW FET and further enhancement with smaller SiNW size could be speculated as attributions of the effect of corners in the cross section of the SiNW channel. As the NWs were fabricated by high temperature thermal oxidation, the corners of the NW have rounded shapes with a curvature radius of $4 \mathrm{~nm}$. Given that the SiNW FETs were fabricated on the same wafer, the peripheral length of the corners can be considered as constant among three devices; only the proportion of the top flat and corner surface within the channel peripheral width changes. Assuming that the top flat surface region performs the same $I_{\mathrm{ON}}$ as the planer SOI device with an $I_{\mathrm{ON}}$ of $324 \mu \mathrm{A} / \mu \mathrm{m}$ for $L_{\mathrm{g}}=190 \mathrm{~nm}$, the drivability of each corner can be extracted as shown in figure 5. The vertical etched surfaces of the channels at both sides are basically (110) surfaces, so that the $\mu_{\text {eff }}$ of the carriers at these surfaces may be degraded compared to the flat surface at the top [10]. However, the effect can be considered as a minor factor in this work as the $h_{\mathrm{NW}}$ is small compared to the $w_{\mathrm{NW}}$. 
TABLE I. BENCHMARK OF MULTI-GATE FETS REPORTED

\begin{tabular}{|c|c|c|c|c|c|c|c|c|c|}
\hline & This work & Ref[11] & Ref[12] & Ref[13] & Ref[14] & Ref[15] & Ref[4] & Ref[16] & Ref[17] \\
\hline NW Cross-sectional shape & Rectangular-like & Rectangular-like & Rectangular-like & Circular & Circular & Elliptical & Elliptical & Fin & Tri-Gate \\
\hline NW Size $(\mathrm{nm})$ & $10 \times 20$ & $10 \times 20$ & \begin{tabular}{|c|}
14 \\
\end{tabular} & \begin{tabular}{l|}
10 \\
\end{tabular} & \begin{tabular}{|l|}
10 \\
\end{tabular} & 12 & $13 \times 20$ & & \\
\hline $\mathrm{L}_{\mathrm{g}}(\mathrm{nm})$ & 65 & 25 & 100 & 30 & 8 & 65 & 35 & 25 & 40 \\
\hline EOT or $T_{o x}(n m)$ & 3 & 1.8 & 1.8 & 2 & 4 & 3 & 1.5 & & \\
\hline $\mathrm{V}_{\mathrm{d}}(\mathrm{V})$ & 1.0 & 1.1 & 1.2 & 1.0 & 1.2 & 1.2 & 1.0 & 1 & 1.1 \\
\hline $\mathrm{I}_{\mathrm{ON}}(\mathrm{uA})$ per wire & 60.1 & 102 & 30.3 & 26.4 & 37.4 & 48.4 & 43.8 & & \\
\hline $\mathrm{I}_{\mathrm{ON}}(\mathrm{uA} / \mathrm{um})$ by circumference & 1609 & 2054 & 430 & 841 & 1191 & 1283 & 825 & 1296 & 1400 \\
\hline $\mathrm{SS}(\mathrm{mV} /$ dec. $)$ & 70 & 79 & 68 & 71 & 75 & $\sim 75$ & 85 & 83 & 76 \\
\hline DIBL $(\mathrm{mV} / \mathrm{V})$ & 62 & 56 & 15 & 13 & 22 & $40-82$ & 65 & 83 & 89 \\
\hline $\mathrm{I}_{\mathrm{ON}} / \mathrm{I}_{\mathrm{OFF}}$ & $\sim 1 \mathrm{E} 6$ & $>1 \mathrm{E} 6$ & $>1 \mathrm{E} 5$ & $\sim 1 \mathrm{E} 6$ & $>1 \mathrm{E} 7$ & $>1 \mathrm{E} 7$ & $\sim 2 \mathrm{E} 5$ & $\sim 3 \mathrm{E} 4$ & $\sim 1 \mathrm{E} 4$ \\
\hline
\end{tabular}

By solving linear equations of the $I_{\mathrm{ON}}$ for the top-flat and corner parts with different SiNW-FETs, a corner with a curvature radius of $4 \mathrm{~nm}$ can exhibit a high $I_{O N}$ of $3000 \mu \mathrm{A} / \mu \mathrm{m}$ can be calculated. On this account, one can calculate that the $80 \%$ of $I_{\mathrm{ON}}$ is dominated at the rounded corner with the smallest device of $h_{\mathrm{NW}}$ and $w_{\mathrm{NW}}$ of 12 and $19 \mathrm{~nm}$, respectively.

\section{DISCUSSION}

Table 1 summarizes the benchmarking of the reported SiNW FETs. Although our device has relatively large $L_{\mathrm{g}}$ and $T_{\text {ox }}$ among the SiNW FETs listed in the table, the 2nd-highest $I_{\mathrm{ON}}$ over $1600 \mu \mathrm{A} / \mu \mathrm{m}$ was demonstrated. One could expect further higher performances with the dimension scaling. For example, with decreasing both the gate length (65 to $32.5 \mathrm{~nm}$ ) and gate oxide thickness ( 3 to $1.5 \mathrm{~nm}$ ) $I_{\mathrm{ON}}$ over $3000 \mu \mathrm{A} / \mu \mathrm{m}$ by circumference could be expected. Among various cross sectional shape of SiNW shown in the table, including rectangle-like, elliptical and circular shapes, one can see a trend that a large $I_{\mathrm{ON}}$ normalized by channel peripheral width is achieved with rectangle-like shape, possibly the effect of rounded corners in the channel.

\section{CONCLUSION}

Very high on-current of gate semi-around SiNW FETs fabricated with conventional CMOS process have been demonstrated. A rectangular-like shape SiNW with a height and a width of 12 and $19 \mathrm{~nm}$, respectively, has revealed a high on-current over $1600 \mu \mathrm{A} / \mu \mathrm{m}$ with a gate length and an oxide thickness of 65 and $3 \mathrm{~nm}$, respectively. The origin of high drivability has been explained by fairly nice effective mobility, and low S/D parasitic resistance as well as the enhancement in the carrier concentration at the rounded surface of SiNW. There is a good possibility that the drivability over $3000 \mu \mathrm{A} / \mu \mathrm{m}$ by circumference could be obtained by future scaling of $L_{\mathrm{g}}$ and EOT even using convention CMOS process.

\section{ACKNOWLEDGEMENT}

The authors thank to Front End Process Program in research dept. 1 and ASKA II line staffs in semiconductor leading edge technologies for kind advice and device fabrication, especially H. Watanabe, Y. Ohji, K. Ikeda, T. Aoyama, Y. Sugita, M. Hayashi, T. Morooka and T. Matsuki. S. S. thanks to Dr. Y. Kobayashi and K. Tsutsui for simulations.

\section{REFERENCES}

[1] Y-K Choi, et al. "Ultrathin-Body SOI MOSFET for Deep-Sub-Ten Micron Era", IEEE Elec. Dev. Lett., vol. 21, no. 5, pp. 254-255, 2000.

[2] J-P. Colinge, "Mutiple-Gate SOI MOSFETs", Solid-State Elec., vol. 48, no. 6, pp. 897-905, 2004.

[3] S. Sato, et al., "High-Performance Si Nanowire FET with a Semi GateAround Structure Suitable for Integration", Proc. of $39^{\text {th }}$ ESSDERC, pp. 249-254, 2009.

[4] S. Bangsaruntip, et al., "High Performance and Highly Uniform GateAll-Around Silicon Nanowire MOSFETs with Wire Size Dependent Scaling", Tech. Dig. of IEDM, pp. 297-300, 2009.

[5] J. G. Fossum, J. -W. Yang, and V. P. Trivedi, "Suppression of Corner Effects in Triple-Gate MOSFETs", IEEE Elec. Dev. Lett., vol. 24, no. 12 , pp. 745-747.

[6] H. Irie and A. Toriumi, "Advanced split-CV technique for accurate extraction of inversion layer mobility in short channel MOSFETs", Extended Abstracts of International Conference of Sol. Stat. Dev. Mat., pp. 864-865, 2005.

[7] K. Endo, et al., "Damage-Free Neutral Beam Etching Technology for High Mobility FinFETs", Tech. Dig. of IEDM, pp 840-843, 2005.

[8] A. Kikuchi, "Phosphorus redistribution during nickel silicide formation”, J. Appl. Phys., vol. 64, no. 2, pp. 938-940, 1988.

[9] M. G. Ancona, and H.F. Tiersten, "Macroscopic physics of the silicon inversion layer", Physical Review B, vol. 4, no. 6, pp. 7959-7965, 1987.

[10] T. Sato et al., "Mobility Anisotropy of Electrons in Inversion Layers on Oxidized Silicon Surfaces", Phys. Rev. B, vol. 4, no. 6, pp. 1950-1960, 1971.

[11] G. Bidal, et al., "High velocity Si-nanodot : A candidate for SRAM applications at $16 \mathrm{~nm}$ node and below", Tech. Dig. of Symp. VLSI Tech., pp. 240-241, 2009.

[12] C. Dupré, et al., " $15 \mathrm{~nm}$-diameter 3D Stacked Nanowires with Independent Gates Operation: $\Phi F E T "$, Tech. Dig, of IEDM, pp. 749$752,2008$.

[13] S. D. Suk, et al., "High Performance $5 \mathrm{~nm}$ radius Twin Silicon Nanowire MOSFET (TSNWFET): fabrication on bulk $\mathrm{Si}$ wafer, characteristics, and reliability", Tech. Dig. of IEDM, pp.717-720, 2005.

[14] Y. Jiang, et al., "Performance Breakthrough in $8 \mathrm{~nm}$ Gate Length GateAil-Around Nanowire Transistors using Metallic Nanowire Contacts", Tech. Dig. of Symp. VLSI Tech., pp. 34-35, 2008.

[15] H. S. Wong, et al., "Gate-all-around Quantum-Wire Field-Effect Transistor with Dopant Segregation at Metal-Semiconductor-Metal heterostructure", Tech. Dig. of Symp. VLSI Tech., pp. 92-93, 2009.

[16] C. Y. Chang, et al., "A 25-nm Gate-Length FinFET Transistor Module for 32nm Node", Tech. Dig. of IEDM, pp.293-296, 2009.

[17] J. Kavalieros, et al., "Tri-Gate Transistor Architecture with High-k Gate Dielectrics, Metal Gates and Strain Engineering", Tech. Dig. of Symp. VLSI Tech., pp.50-51, 2006. 